\section{PTU-071 SUPPORTING PATIENTS WITH PERCUTANEOUS ENDOSCOPIC GASTROSTOMY (PEG) IN THE COMMUNITY: DEVELOPING A HOME ENTERAL FEED PROGRAMME TO AVOID HOSPITAL READMISSIONS}

doi:10.1136/gut.2011.239301.199

SWhite, ${ }^{1 *}$ MKurien, ${ }^{2}$ GSimpson, ${ }^{1} \mathrm{JGrant},{ }^{2}$ DS Sanders, ${ }^{2}$ MEMcAlindon ${ }^{2}$ Department of Dietetics, Royal Hallamshire Hospital, Sheffield, UK; ${ }^{2}$ Department of Gastroenterology, Royal Hallamshire Hospital, Sheffield, UK

Introduction Although patients who have a PEG inserted are often discharged to a complex network of medical and social support on discharge, PEG-related complications are a common cause of hospital readmission. ${ }^{1}$

Methods In Sheffield, PEG aftercare within the community is provided by two home enteral feed (HEF) dietitians and a dietetic assistant. They provide support and training to patients, carers and other healthcare professionals. Data regarding their activity is prospectively entered into an Infoflex database. We aimed to determine the benefits of a dedicated PEG aftercare service and also the impact that this service can have on reducing hospital readmission rates following PEG insertion.

Results 280 patients in Sheffield were receiving enteral feeding at home between April 2009 and April 2010. 85 (30\%) were new patients, with 79 (93\%) being reviewed within 5 days of hospital discharge. 2237 referrals were received by the HEF team regarding these 280 patients. Services provided by the HEF team included: home visits ( $\mathrm{n}=1019)$, nursing and residential home reviews $(n=525)$, telephone advice $(n=683)$ to patients, carers or healthcare professionals and ten hospital assessments. There were 343 PEG-related complications seen be the HEF team during this period. The commonest problems were over-granulated stoma sites $(30 \%)$, broken $\mathrm{Y}$ adaptors $(21 \%)$, tube blockages $(14 \%)$, and bumper related problems (14\%). 103 patients required new balloon gastrostomies, of which $56(43 \%)$ were performed as an emergency procedure. 228 hospital admissions were definitely avoided due to direct actions taken by the HEF team. While sixty home enterally fed patients were admitted to hospital during this period, only $8(13 \%)$ of these were admitted due to PEG related problems. These admissions occurred during periods when the HEF team were unavailable or when local community PEG protocols were not followed.

Conclusion While patients with PEGs may need attention to a variety of complex medical problems, the vast majority encounter problems specifically related to their PEGs after discharge. HEF Dietitians trained in PEG care reduce unnecessary hospital readmissions. This is achieved by repairing and replacing PEGs in the community, but also by underpinning the service with a training programme designed to allow patients, carers and other professionals to do the same.

Competing interests None.

Keywords None.

\section{REFERENCE}

1. Sanders DS, Carter MJ, D’Silva J, et al. Percutaneous endoscopic gastrostomy: a prospective analysis of hospital support required and complications following discharge to the community. Eur J Clin Nutr 2001;55(7):610-14. 